\title{
Subcutaneous Emphysema: A Case Report of an Atypical Manifestation of PCP in an HIV Patient
}

\author{
Drew Kotler, MD and Drew Johnson, MD
}

\section{INTRODUCTION}

Pneumocystis pneumonia (PCP), caused by Pneumocystis jirovecii (P. jirovecii), is a fungal opportunistic infection seen in poorly controlled Human Immunodeficiency Virus (HIV) and is considered an Acquired Immune Deficiency Syndrome (AIDS) defining illness. It is also found in other immunocompromised states, such as hyper IgM syndrome and in patients on immunosuppressive therapy. In the HIV+ population, a CD4+ T-lymphocyte count (CD4 count) less than 200 cells $/ \mathrm{mm}^{3}$ is associated with an increased risk of PCP. Combination antiretroviral therapy in conjunction with PCP prophylaxis has significantly decreased the prevalence of this opportunistic infection. In the United States HIV-infected population, PCP prevalence has decreased from 29.9 per 1000 person years between 1994 to 1997 to 3.9 per 1000 person years between 2003 to 2007. ${ }^{1}$ At the onset of the HIV/AIDS epidemic, PCP occurred in $70 \%$ to $80 \%$ of patients with AIDS. ${ }^{2}$ PCP should be highly suspected in HIV+ patients with bilateral symmetrical interstitial infiltrates on chest radiography, hypoxemia, and a CD4 count less than 200 cells $/ \mathrm{mm}^{3}$ in addition to infectious respiratory symptoms such as cough, fever, and dyspnea. For definitive diagnosis, histopathologic or cytopathologic organism identification is required, most commonly via bronchoalveolar lavage or tissue biopsy. In addition to pneumonia, P. jirovecii can invade the liver, spleen, and kidneys. It has also been associated with pneumothorax. Less commonly, it has been associated with pneumomediastinum without pneumothorax

\section{CASE PRESENTATION}

This is a 39-year-old man with a twenty-year history of HIV who presented with one month of worsening shortness of breath, dry cough, and dysphagia. At admission, the patient had a CD4 count of 52 cells $/ \mathrm{mm}^{3}$, a viral load of 456,074 copies $/ \mathrm{mL}$, and a history of non-adherence to HIV treatment. Prior to admission, the patient was not taking his prescribed antiretroviral therapy or PCP prophylaxis. These were re-initiated at the time of admission. In the hospital, the patient had progressive hypoxia that eventually required high flow oxygen supplementation at $35 \mathrm{~L} /$ minute and $100 \% \mathrm{FiO}_{2}$. Initial chest $x$-ray showed diffuse bilateral infiltrates.
Thorax CT demonstrated a "crazy-paving" pattern with overlapping of thickened septa and diffuse ground glass opacities. Based on the clinical presentation, imaging, and consultation with the Pulmonary and Infectious Diseases teams, the patient was diagnosed with likely PCP. He declined confirmatory lavage, bronchoscopy or surgical lung biopsy. He was started on IV trimethoprimsulfamethoxazole and methylprednisolone for treatment of PCP. He was also given one week of vancomycin and piperacillin-tazobactam for empiric treatment of bacterial pneumonia. Other infectious etiologies, including mycobacterial infection, were ruled out. Acid Fast Bacilli (AFB) cultures of sputum and serum were negative. A respiratory viral pathogen PCR panel was negative. Blood and urine cultures were unrevealing. Additionally, serum cryptococcal antigen was negative. Later in the patient's hospitalization, he acutely developed significant facial swelling. Due to concern for allergic angioedema and possible airway compromise, otolaryngology performed a bedside nasopharyngolaryngoscopy. The airway was patent without visible edema. Physical exam was notable for crepitus of the chest, neck, and face. $X$-rays were obtained and confirmed the presence of subcutaneous emphysema (figure 1). CT of the chest showed pneumomediastinum extending into the neck (figure 2). Esophageal perforation was ruled out with a swallow study using video fluoroscopy. The patient was evaluated by thoracic surgery, but there was no surgical intervention required. He was monitored with serial imaging.

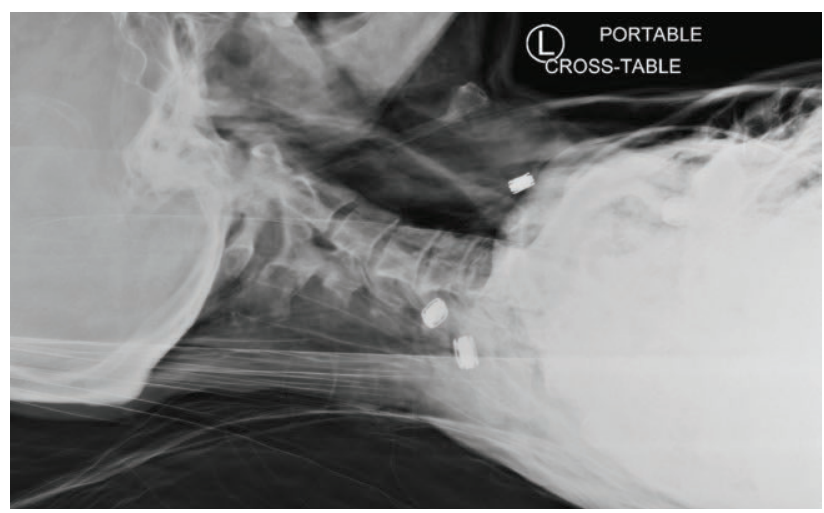

Figure 1. X-ray demonstrating subcutaneous emphysema of the neck and face. 


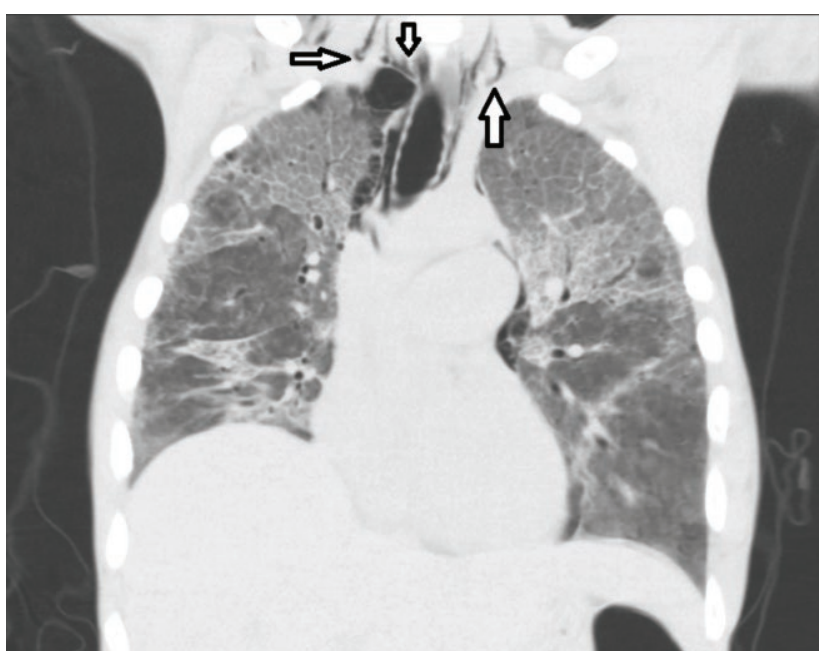

Figure 2. CT demonstrating subcutaneous emphysema of the neck and pneumomediastinum.

\section{OUTCOME}

The pneumomediastinum was likely secondary to PCP after the formation of pneumatoceles (figure 3). Its progression to subcutaneous emphysema of the neck and face was likely exacerbated by the high flow oxygenation. For the subcutaneous emphysema, the patient was offered treatment with imaging-guided placement of a pigtail catheter, which he declined. Despite a prolonged treatment course with IV trimethoprim-sulfamethoxazole and methylprednisolone, the patient still required high flow oxygen supplementation. Given his poor prognosis with HIV/ AIDS and lack of improvement, the patient created a do not resuscitate advance directive and was discharged to an inpatient hospice facility with high flow oxygen supplementation at $35 \mathrm{~L} /$ minute and $50 \% \mathrm{FiO}_{2}$ for adequate oxygenation. The patient died less than a month after discharge.

\section{DIFFERENTIAL DIAGNOSIS}

In HIV patients, PCP remains one of the most common opportunistic infections. However, the differential for this patient must remain broad. Bacterial, mycobacterial, viral, and fungal pneumonias should be considered. Possible non-infectious etiologies include neoplasm, drug hypersensitivity, and pulmonary hypertension. Acute Interstitial Pneumonitis (AIP), Pulmonary Veno-occlusive Disease (PVOD) and Acute Respiratory Distress Syndrome (ARDS) were also on the differential for this patient. Due to high clinical suspicion, empiric PCP treatment was initiated while the work-up was still underway. The patient declined right heart catheterization to rule out pulmonary hypertension.

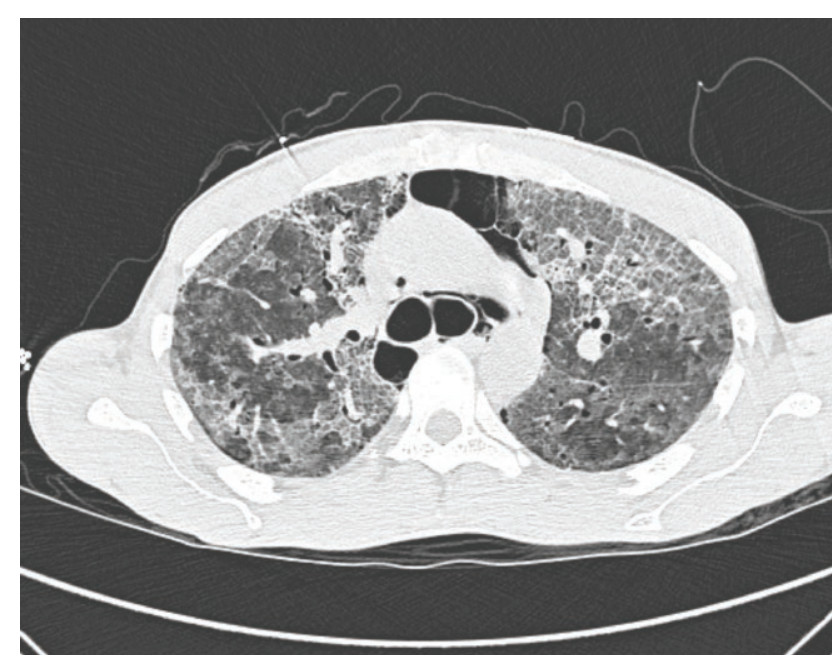

Figure 3. CT demonstrating pneumatoceles

\section{DISCUSSION}

Since PCP has a high mortality rate if left untreated, patients are often empirically started on treatment for PCP if they present with a pulmonary infection in the setting of HIV infection and a CD4 count less than 200 cells $/ \mathrm{mm}^{3}$. Trimethoprim-sulfamethoxazole remains the first-line treatment, with or without steroids depending on the alveolar-arterial gradient. In addition to the complications inherent to pneumonia, patients with PCP are at increased risk of developing air-filled cystic spaces known as pneumatoceles. These pneumatoceles are at risk of rupturing and allowing air to spread along the peribronchial lining and fascial planes of the chest, neck, and axilla. This can give rise to pneumomediastinum, subcutaneous emphysema, and in severe cases, pneumorrhachis (air within the spinal canal). ${ }^{3}$ PCP can also be complicated by the development of a pneumothorax. The prevalence of pneumomediastinum in PCP is thought to be $0.4-3.3 \% .{ }^{4}$ The abrupt change in transalveolar pressure that leads to spontaneous pneumomediastinum was first described in 1939 by Macklin who proposed that an abrupt change in pressure due to coughing or straining could cause alveolar rupture and tracking of air along the interstitial sheaths toward the mediastinum. ${ }^{5}$ In general, alveolar rupture is reported to be the cause of spontaneous pneumomediastinum in over $95 \%$ of cases. Esophageal and tracheal disruptions are less common but more alarming causes. Compared to healthy individuals with no underlying infection, patients with pneumomediastinum secondary to PCP pneumonia have a worse prognosis. ${ }^{5}$ While $x$-ray is often adequate, CT scan is the gold standard for radiographic diagnosis. Complications are usually self-limited, and may only require conservative 
management and observation, in addition to antimicrobial therapy directed at the underlying infection. Several case reports in the literature describe pneumomediastinum at presentation of PCP, but it may be seen any time during the clinical course of the disease..$^{6,7}$

\section{KEY POINTS}

This case represents a classic presentation of PCP in an HIV patient with a CD4 count of less than 200 cells $/ \mathrm{mm}^{3}$. The patient had hypoxia, non-productive cough, and bilateral diffuse infiltrates on chest radiography. These findings justify the initiation of empiric treatment with IV trimethoprim-sulfamethoxazole and methylprednisolone. Furthermore, pneumomediastinum is more likely to be found in PCP compared to other causes of pneumonia. While more rare, the development of subcutaneous emphysema of the neck and face is consistent with pneumomediastinum secondary to PCP. Additionally, this case reinforces the fact that while angioedema is a common cause of facial swelling, there are other etiologies that should be considered. Lastly, the severity of this patient's illness and profound hypoxia requiring high amounts of supplemental oxygen underscores the importance of antiretroviral therapy and PCP prophylaxis.

\section{REFERENCES}

1. Buchacz, Kate, et al. "AIDS-defining opportunistic illnesses in US patients, 1994-2007: a cohort study." Aids 24.10 (2010): 1549-1559.

2. Phair, John, et al. "The risk of Pneumocystis carinii pneumonia among men infected with human immunodeficiency virus type 1." New England Journal of Medicine 322.3 (1990): 161-165

3. Jung, Hanna, et al. "Spontaneous pneumomediastinum with concurrent pneumorrhachis." Korean Journal of Thoracic and Cardiovascular Surgery 47.6 (2014): 569-571.

4. Cheng, Wan-Ling, et al. "Pneumomediastinum in patients with AIDS: a case report and literature review." International Journal of Infectious Diseases 22 (2014): 31-34

5. Sakai M, Murayama S, Gibo M, Akamine T, Nagata O. Frequent cause of the Macklin effect in spontaneous pneumomediastinum: demonstration by multidetector-row computed tomography. $]$ Comput Assist Tomogr. 2006:30(1):92-94

6. Moss, Sheila, Peter B. Carey, and C. R. Hind. "Pneumocystis carinii pneumonia presenting with pneumomediastinum in an HIV-positive patient." Postgraduate medical journal 71.832 (1995): 96.

7. Vilalona-Calero, Miguel A., Stanley S. Schrem, and Kenneth R. Phelps. "Pneumomediastinum complicating Pneumocystis carinii pneumonia in a patient with AIDS." The American journal of the medical sciences 297.5 (1989): $328-330$ 


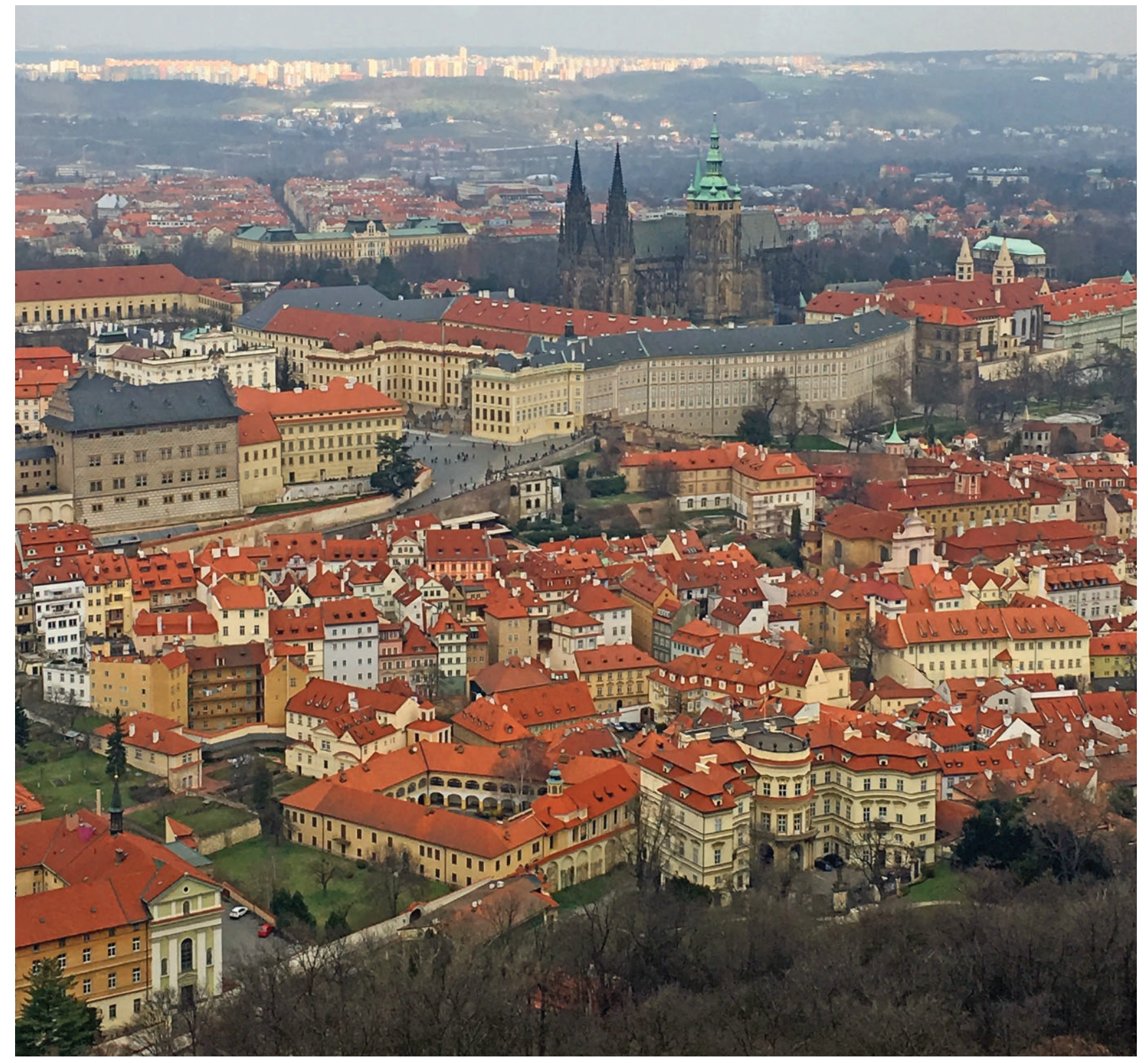

Jessica Dahmus, MD 